\title{
Montmorency Tart cherries (Prunus cerasus L.) modulate vascular function acutely, in the absence of improvement in cognitive performance
}

\author{
K. M. Keane ${ }^{1 *}$, C. F. Haskell-Ramsay ${ }^{2}$, R. C. Veasey ${ }^{2}$ and G. Howatson ${ }^{1,3}$ \\ ${ }^{1}$ Department of Sport, Exercise and Rehabilitation, Faculty of Health and Life Sciences, Northumbria University, \\ Newcastle Upon Tyne NE1 8ST, UK \\ ${ }^{2}$ Brain, Performance and Nutrition Research Centre, Faculty of Health and Life Sciences, Northumbria University, \\ Newcastle upon Tyne NE1 8ST, UK \\ ${ }^{3}$ Water Research Group, School of Environmental Sciences and Development, Northwest University, Potchefstroom 2531, \\ South Africa
}

(Submitted 13 July 2016 - Final revision received 18 October 2016 - Accepted 7 November 2016 - First published online 19 December 2016)

\section{Abstract}

Cerebral blood volume and metabolism of oxygen decline as part of human ageing, and this has been previously shown to be related to cognitive decline. There is some evidence to suggest that polyphenol-rich foods can play an important role in delaying the onset or halting the progression of age-related health disorders such as CVD and Alzheimer's disease and to improve cognitive function. In the present study, an acute, placebo-controlled, double-blinded, cross-over, randomised Latin-square design study with a washout period of at least $14 \mathrm{~d}$ was conducted on twenty-seven, middle-aged (defined as 45-60 years) volunteers. Participants received either a $60 \mathrm{ml}$ dose of Montmorency tart cherry concentrate (MC), which contained 68.0 (SD 0.26) mg cyanidin-3-glucoside/1, 160.75 (SD 0.55) mean gallic acid equivalent/1 and 0.59 (sD 0.02) mean Trolox equivalent/1, respectively, or a placebo. Cerebrovascular responses, cognitive performance and blood pressure were assessed at baseline and 1,2, 3 and $5 \mathrm{~h}$ following consumption. There were significant differences in concentrations of total $\mathrm{Hb}$ and oxygenated $\mathrm{Hb}$ during the task period $1 \mathrm{~h}$ after $\mathrm{MC}$ consumption $(P \leq 0 \cdot 05)$. Furthermore, MC consumption significantly lowered systolic blood pressure $(P \leq 0.05)$ over a period of $3 \mathrm{~h}$, with peak reductions of $6 \pm 2 \mathrm{mmHg}$ at $1 \mathrm{~h}$ after $\mathrm{MC}$ consumption relative to the placebo. Cognitive function and mood were not affected. These results show that a single dose of MC concentrate can modulate certain variables of vascular function; however, this does not translate to improvements in cognition or mood.

Key words: Montmorency tart cherries: Cerebral blood flow: Blood pressure: Cognitive performance

Montmorency tart cherries (L. Prunus cerasus; MC) and their derivatives are functional foods that are high in numerous phytochemicals $^{(1-5)}$ including flavonoids such as isorhamnetin, kaempferol, quercetin, catechin, epicatechin, procyanidins and anthocyanins $^{(6,7)}$. It has been previously shown that MC attenuate inflammation ${ }^{(1)}$ and oxidative stress $^{(8,9)}$ as well as improve aspects of vascular function ${ }^{(3)}$. A property underlying the potential vascular effects of tart cherries is an ability to modulate blood flow parameters. Cherry extracts have been shown, in cell and animal models, to exert a range of cardioprotective effects that include increasing nitric oxide production and antioxidant status, reducing lipid oxidation and inhibiting inflammatory pathways ${ }^{(1,4)}$. Even more recently, Keane et al. ${ }^{(3)}$ demonstrated an increase in plasma phenolic acids (vanillic and protocatechuic) following MC consumption in humans; these compounds were also shown to modulate vascular smooth muscle cell behaviour in vitro. In a subsequent study, Keane et al. demonstrated that circulating phenolic metabolites derived from Montmorency tart cherry juice are, at least in part, responsible for an acute reduction in systolic blood pressure (SBP) in men with early hypertension ${ }^{(10)}$.

Ageing is associated with deficits in motor function, which include decreases in balance, muscle strength, coordination and cognitive function, especially in tasks that require the use of spatial learning and memory. This has been suggested to be caused by a concurrent decline in cerebral blood volume and metabolism of oxygen, which also occurs as a result of ageing ${ }^{(11)}$. These decrements have been reported in numerous studies in both animals ${ }^{(12,13)}$ and humans ${ }^{(14,15)}$. A large number of dietary interventions using polyphenol-rich foods or beverages, particularly those using tea ${ }^{(16)}$, Ginkgo Biloba ${ }^{(17)}, \operatorname{cocoa}^{(18)}$ and blueberry ${ }^{(19)}$, have demonstrated beneficial effects on memory

Abbreviations: BP, blood pressure; CBF, cerebral blood flow; deoxy-Hb, deoxygenated Hb; DV, digit vigilance; MC, Montmorency tart cherry; NIRS, near-IR spectroscopy; oxy-Hb, oxygenated Hb; PLA, placebo; RVIP, rapid visual information processing; SBP, systolic blood pressure; WBB, wild blueberry.

* Corresponding author: K. Keane, email k.keane@northumbria.ac.uk 
and learning in both animals and humans. Although it is not clear whether tart cherries can decrease the risk of neurodegenerative ageing or diseases such as Parkinson's and Alzheimer in humans, studies with animal models are more positive and suggest that the phenolic compounds found in tart cherries may exert their beneficial effects through their ability to lower oxidative stress and anti-inflammatory properties or by altering directly the signalling involved in neuronal communication, Ca buffering ability and stress signalling pathways among others ${ }^{(19,20)}$.

Seymour et $a l .{ }^{(21)}$ showed that intake of $1 \%$ tart cherry diet significantly reduced stroke-related phenotypes in rats. Tart cherry intake also reduced brain NF- $\kappa \mathrm{B}$ activity and the related pro-inflammatory transcripts. Interestingly, in 2015, Kirakosyan et $a{ }^{(22)}$ confirmed that tart cherry anthocyanins cross the blood-brain barrier. In a more recent addition to the literature, thirty, 19-month-old, male Fischer 344 rats that received either a control diet or a diet supplemented with $2 \%$ MC for 6 weeks were examined. Their results showed that, although there were no changes in motor performance, tart cherry supplementation significantly improved working memory of aged rats ${ }^{(23)}$. However, there is a paucity of data from human trials to extrapolate these findings to hominids.

Caldwell et al. ${ }^{(24)}$ previously demonstrated that, regardless of dose, cherry juice had no acute impact on cognitive function in young people, older people or dementia patients. They concluded that, although cherry juice may have an acute impact on cardiovascular function, there was no change in cognitive performance $6 \mathrm{~h}$ after consumption. In contrast, a chronic supplementation study ${ }^{(25)}$ reported that daily consumption of sweet cherries for 12 weeks improved cognitive performance across almost all tasks in older adults with mild-to-moderate dementia; this group showed improvements in categories such as verbal fluency and tasks related to verbal learning and memory, and concluded that the positive changes have clinical relevance for these cognitive improvements. It would therefore appear that the cerebrovascular response required to elicit measurable changes in cognitive function can only be achieved with longer-term dosing strategies ${ }^{(26)}$. Contrary to this theory, two recent additions to the literature suggest that acute supplementation has the ability to improve aspects of cognitive function. Acute blackcurrant supplementation was shown to improve both digit vigilance (DV) and rapid visual information processing (RVIP) in healthy younger humans ${ }^{(27)}$. Similarly, acute wild blueberry (WBB) supplementation was shown to improve final immediate recall, delayed word recognition and accuracy on cognitively demanding incongruent trials in the interference task in children ${ }^{(28)}$. Therefore, it is possible that Caldwell et al. reported no impact of cherry supplementation on cognitive function as they used sweet cherries as an intervention. It has previously been speculated that sweet cherries are not as rich in phytochemical compounds as tart cherries ${ }^{(6)}$.

Polyphenol-rich foods have also been reported to improve cerebral haemodynamics assessed by near-IR spectroscopy (NIRS) and functional MRI (fMRI). Wightman et al. ${ }^{(29)}$ assessed the effect of epigallocatechin gallate (EGCG) on cerebral blood flow (CBF) using NIRS in healthy adults. Their results suggested that $135 \mathrm{mg}$ of EGCG caused a reduction in total $\mathrm{Hb}$, a proxy for $\mathrm{CBF}$ during cognitive tasks relative to the placebo. Changes in
CBF have also been demonstrated following resveratrol ${ }^{(30)}$ and beetroot supplementation ${ }^{(31)}$. Krikorian et al. ${ }^{(32)}$ used fMRI to examine the effect of Concord grape juice on neurocognitive function. In total, sixteen adults aged $>68$ years with mild agerelated memory decline were supplemented with either a grape juice (444 ml average) containing, on average, $209 \mathrm{mg}$ of polyphenols or a sugar-matched placebo for 16 weeks. Results found that after 16 weeks there were reductions in semantic interference on memory tasks and relatively greater activation in the anterior and posterior regions of the right hemisphere in the grape juice-treated group. Similarly, people with mild memory complaints, who drank pomegranate juice daily, performed better on memory task compared with placebo and displayed an increase in brain activation measured by $\mathrm{fMRI}^{(33)}$. Very little has been reported on the effect of acute polyphenol supplementation on cerebral haemodynamics, with the majority of this work carried out with flavanol-rich $\operatorname{cocoa}^{(34,35)}$. At present, no attempt has been made to examine the haemodynamic response to acute tart cherry supplementation.

Notwithstanding, given that MC are capable of modulating human vascular function (particularly in relation to blood pressure (BP) and vascular smooth muscle behaviour), we hypothesised that CBF could also be acutely modulated, and thus consequently improve cognitive performance in humans. Therefore, the aim of the present study was to assess the impact of Montmorency tart cherry juice consumption on prefrontal cortical haemodynamics, cognitive function and BP in middleaged adults.

\section{Methods}

\section{Participants}

In total, thirty, healthy, middle-aged (defined as 45-60 years) adults (ten female, twenty male, twenty-eight right-handed, two left-handed adults) were recruited to take part in the study; the mean age, stature, mass and BMI were 50 (SD 6) years, 170.7 (SD 9.1) cm, 76.0 (SD 16.0) kg and 26.1 (SD 4.9) kg/m², respectively. All participants were in apparent good health as assessed by a health-screening questionnaire. This questionnaire was administered to highlight any contraindications to taking part in the study. Exclusion criteria included those who had suffered a head injury, neurological disorder or neurodevelopmental disorder. In addition, those who had any relevant food allergies or intolerances, smoked tobacco, drank excessive amounts of caffeine ( $>6$ cups coffee/d ( $>450 \mathrm{mg}$ caffeine/d)) or took illicit social drugs were also identified as contraindications to participation. All exclusion criteria were self-reported. The study was conducted in accordance with the Declaration of Helsinki and ratified by the University's Research Ethics Committee. All enrolled participants provided their written informed consent. This study was registered as a clinical trial with clinicaltrials.gov (NCT02381860).

\section{Study design}

This study used a placebo-controlled, double-blinded, crossover, randomised Latin-square design with two experimental 
arms and a washout period of at least $14 \mathrm{~d}$ (mean 15 (SD 2) d); participants were randomly allocated to receive a $60-\mathrm{ml}$ dose of MC concentrate or a placebo (PLA). In all, fourteen participants received the $\mathrm{MC}$ concentrate on the first visit, with the remainder receiving the PLA. A washout of at least $14 \mathrm{~d}$ was chosen on the basis of previous literature suggesting that these phenolic compounds are quickly absorbed and/or excreted $^{(3,10,36)}$. Each participant was required to attend the laboratory on three separate occasions. Each visit was at the same time of day (within participant) and was preceded by an overnight fast ( $\geq 10 \mathrm{~h}$ ). The first visit was an initial screening and familiarisation visit, during which participants were screened with regard to the study exclusion/inclusion criteria, briefed with regard to compliance requirements, provided their written informed consent, and were given full training and familiarised to the cognitive tasks. On the subsequent experimental days, participants reported to the laboratory between 07.00 and 09.00 hours, and a baseline BP reading was taken. This was followed by a baseline cognitive assessment and CBF measures by NIRS and transcranial Doppler. Participants then consumed the intervention beverage (either MC or PLA), following which they sat quietly watching one of a selection of non-arousing digital versatile disc (DVD), during a 1-h 'absorption period'. Subsequent cognitive assessments and blood flow measures were taken 1, 2, 3 and $5 \mathrm{~h}$ after consumption; $\mathrm{BP}$ was measured hourly. Between cognitive test sessions, participants continued to watch a selection of non-arousing DVD. No additional food or fluid was provided during the study period except for low-nitrate mineral water, which was provided ad libitum. The total volume of water consumed on the first experimental day was recorded and participants consumed the same volume on the second visit. The reason for this was to accurately examine the efficacy of the intervention.

\section{Treatments and dietary control}

The MC concentrate (CherryActive) was stored at $4{ }^{\circ} \mathrm{C}$ before use. Participants consumed either $60 \mathrm{ml}$ of $\mathrm{MC}$ concentrate (which according to the manufacturer is estimated to be equivalent to about 180 whole cherries) or fruit-flavoured cordial in a double-blinded, cross-over manner. This estimate was based on the Brix value of sucrose in $100 \mathrm{~g}$ of solution. The decision to use $60 \mathrm{ml}$ was based on previous studies that showed a greater uptake of anthocyanin and phenolic acids in vivo after consumption when compared with a 30-ml dose $\mathrm{e}^{(2,3)}$. In addition, this study identified that of the three MC analogues studied (frozen, dried and concentrated), the MC concentrate had the greatest antioxidant activity as well as total anthocyanin and phenolic content ${ }^{(3)}$. The MC concentrate was examined for total anthocyanins, total phenolic content and Trolox equivalent antioxidant capacity using techniques previously described by Keane et $a l^{(10)}$. The MC concentrate was found to contain 68.0 (sD 0.26) mg cyanidin-3-glucoside/l, 160.75 (sD 0.55) mean gallic acid equivalent/l and 0.59 (SD 0.02) mean Trolox equivalent/l, respectively. The concentrate was diluted with $100 \mathrm{ml}$ of water before consumption.

The PLA supplement consisted of a commercially available, low fruit $(<1 \%)$ cordial (Kia Ora, Coca Cola Enterprises) mixed with water, whey protein isolate (Arla Foods Ltd) and maltodextrin (MyProtein Ltd), to match the MC concentrate for volume and macronutrient content (energy $=854 \mathrm{~kJ}(204 \mathrm{kcal})$, volume $=60 \mathrm{ml}, \quad$ carbohydrates $=49 \mathrm{~g}, \quad$ protein $=2.2 \mathrm{~g}$ and fat $=0 \mathrm{~g}$ ). The total anthocyanin content (used for colour purposes only) and total antioxidant capacity of the PLA were lower than the limits of detection, with trace amounts of phenolics (8.26 (sD 0.04) mean gallic acid equivalent/1). All drinks were prepared and all bottles were covered in tape before the study by a third party. Before study commencement, it was explained to participants that the aim of the study was to investigate the effect of a fruit juice on vascular function; therefore, they were unaware which beverage was the experimental drink. Participants were instructed to follow a low-phenolic diet for $48 \mathrm{~h}$ before each arm of the trial by avoiding fruits, vegetables, tea, coffee, alcohol, chocolate, cereals, wholemeal bread, grains and spices and were asked to refrain from strenuous exercise. Compliance with the dietary restrictions was assessed with a standardised, self-reported 2-d dietary record. All participants complied with the low-phenolic diet, and this was confirmed via visual inspection of the food diaries.

\section{Cognitive tasks}

All cognitive and mood measures were delivered using the Computerised Mental Performance Assessment System (COMPASS, Northumbria University), a purpose-designed software application for the flexible delivery of randomly generated parallel versions of standard and novel cognitive assessment tasks. This assessment system has previously been shown to be sensitive to nutritional interventions following both acute ${ }^{(37)}-$ including acute supplementation with phenolics ${ }^{(27)}$ - and chronic supplementation ${ }^{(38)}$. At each of the aforementioned time points, a cognitive assessment test was completed. This assessment was a collection of three tasks that lasted $9 \mathrm{~min}$, and was performed twice, which equated to $18 \mathrm{~min}$ in total. This was followed by a series of visual analogue scales to assess perceptions of fatigue and difficulty. The types of tests chosen have been previously used to detect changes in cognitive function following nutritional interventions ${ }^{(18,27,30)}$. In order to assess the relationship between specific brain regions and any changes in $\mathrm{CBF}$, a selection of tasks that engender either higher or lower activation of the frontal cortex was used. The low activation' tasks comprised of a sustained attention test (DV). The 'high activation' tasks (RVIP and Stroop tasks) entailed a higher cognitive workload and have been shown to increase activity in the prefrontal cortex ${ }^{(39,40)}$. The battery of cognitive tasks is described in more detail below.

Digit vigilance. The DV task is a measure of sustained attention and psychomotor speed ${ }^{(41)}$. A single target digit was randomly selected and constantly displayed on the right-hand side of the screen. A series of single digits appeared on the left-hand side of the screen, one at a time, at the rate of 150 per minute. The participant was required to press the target button on the response pad as quickly as possible every time the digit in the series matched the target digit. The task lasted $3 \mathrm{~min}$ in total. 
Task outcomes included accuracy (\%) and reaction time for correct responses (ms).

Rapid visual information processing. The RVIP task is a measure of sustained attention and working memory ${ }^{(41)}$. This task requires the participant to monitor a continuous series of single digits for targets of three consecutive odd or three consecutive even digits. The digits are presented on the computer screen one at a time at the rate of $100 / \mathrm{min}$ in pseudo-random order, and the participant responds to the detection of a target string by pressing the target button on the response pad as quickly as possible. The task lasted $3 \mathrm{~min}$ in total. Task outcomes included number of target strings correctly detected (\%) and average reaction time for correct detections (ms).

Stroop. The Stroop test is a measure of attention, inhibition and cognitive flexibility ${ }^{(42)}$. In this task, participants were presented with a colour name. The colour name presented was written in a coloured font, either the same 'congruent' or a different 'incongruent' font. Participants had to identify the colour of the font the word was written in, rather than the colour that the word was describing, via a response pad with coloured keys. Participants were presented with ninety stimuli in total, taking about 3 min to complete. Task outcomes included the number of correct responses (\%) and the average response time for congruent and incongruent stimuli (ms).

Visual analogue scales. Participants were required to rate how 'alert', 'concentrated' and 'mentally fatigued' they felt and how 'difficult' they had found the tasks after each cognitive assessment repetition by indicating on a 100-mm line with the cursor ('not at all' at one end of the line and 'extremely' at the other end) for alertness, fatigue and level of difficulty as well as ('very low' to 'very high') for concentration. The VAS were scored as \% along the line denoting more of the relevant adjective.

\section{Blood pressure}

$\mathrm{BP}$ was measured using a non-invasive digital automatic BP monitor (M10-IT; Omron Healthcare). The BP cuff was fitted by the same researcher at each of the six time points. The interand intra-trial \%CV for this method was 4.2 and $1.3 \%$, respectively.

\section{Cerebrovascular responses}

Transcranial Doppler imaging. Cerebral blood flow velocity (CBFV) in the middle cerebral artery was determined using transcranial Doppler sonography (Doppler-Box, Compumedics DWL). A 2-MHz Doppler probe was positioned over the rightmiddle cerebral artery, using previously described search techniques $^{\text {(43) }}$, and secured with an adjustable headset (DiaMon; Compumedics DWL). The mean depth for Doppler signals was $62(\mathrm{sD} 3) \mathrm{mm}$. All data were sampled at $200 \mathrm{~Hz}$ (PowerLab 16/30; ADInstruments Ltd) and processed offline (LabChart version 5.4.2; ADInstruments Ltd).
Near-IR spectroscopy. The NIRS is a non-invasive brain imaging technique in which two nominal wavelengths of light, which are differentially absorbed by oxygenated (oxy-Hb) and deoxygenated $\mathrm{Hb}$ (deoxy- $\mathrm{Hb}$ ), respectively, are introduced through the skull via a laser emitter. They are then measured, following transit through the upper surface of the cortex, by an optode placed at a pre-set distance from the light source. NIRS has been used extensively as a technique for multiple-channel imaging of task-related brain activity over relevant areas of the head, including groups suffering from potential declines in $\mathrm{CBF}^{(44)}$. In the present study, cerebral oxygenation was assessed using NIRS (NIRO-200NX; Hamamatsu Photonics K.K.). Two near-IR sensors were placed over the left and right frontal lobe region of the forehead corresponding to the international 10-20 system Fp1 and Fp2 EEG positions; these signals were averaged to determine cerebral oxygenation. The sensors were secured to the skin using double-sided adhesive tapes and shielded from ambient light using an elastic bandage. The sensors alternately emit two wavelengths of near-IR light (approximately 765 and $855 \mathrm{~nm}$ ) with an emitter/optode separation distance of $4 \mathrm{~cm}$. The NIRS data were acquired continuously every $5 \mathrm{~s}$ and recorded for later offline analysis. The NIRS data output was time stamped at the start of each task segment to assure that data corresponded to the relevant period of task performance. Relative concentration changes in oxy-Hb, deoxy$\mathrm{Hb}$ and total $\mathrm{Hb}$ were calculated.

\section{Statistical analysis}

Cognitive performance, $\mathrm{BP}$ and $\mathrm{CBFV}$ data were analysed using a treatment $\times$ time point mixed model ANOVA. Mauchly's Test of Sphericity was used to check homogeneity of variance for all ANOVA analyses; where necessary, violations of the assumption were corrected using the Greenhouse-Geisser adjustment. Significant main effects were followed-up using Šidák post hoc analysis. The analysis of NIRS data was conducted with Minitab 15 for Windows (Minitab Inc.). Before the primary analysis, a within-subjects ANOVA was carried out with left/right optode included as a factor (hemisphere $\times$ treatment group) for each task. As there were no treatment-related interactions involving hemisphere, data from the two channels were averaged across hemispheres for the analysis and figures reported below. For each variable (oxy-Hb, deoxy-Hb and total $\mathrm{Hb}$ ), data were converted to 'change from baseline' (calculated from baseline pre-treatment period). Task length was fixed for the DV (180s) and RVIP (180s), but NIRS data from the Stroop test were truncated so that the same amount of data was analysed for all participants during each task period. Data from the 'resting/absorption' period (minutes 1-60) and the task performance were analysed separately for all time points (pre-supplement, 1, 2, 3 and 5h). Data from the 'resting/ absorption' period were averaged across six equal 10-min epochs and analysed by two-way repeated-measures ANOVA (epoch $\times$ treatment). Data from the task period were averaged across six equal 3-min epochs. These data were analysed by a three-way repeated-measures ANOVA (task (epoch) $\times$ treatment $\times$ time point). 
In the absence of any directly relevant data, it was suggested that a sample size of twenty-four would be adequate to have greater than $80 \%$ chance of detecting the medium effect sizes demonstrated in previous studies assessing the effect of polyphenols on NIRS parameters ${ }^{(45)}$. The resultant sample size of twenty-seven (for a within-subject, cross-over design) was in excess of the typical sample sizes for NIRS investigations.

\section{Results}

In total, thirty male and female participants volunteered to take part in the present study, but three participants voluntarily withdrew after the first study day because of time constraints ( $n$ 27). There were no adverse events reported in response to the intervention products. All participants complied with the low-polyphenolic diet according to the food diaries.

\section{Cognitive performance and mood}

No significant treatment-related differences were observed for any of the cognitive or mood measures $(P>0.05)$. The absolute values for task scores and mood ratings are given in Tables 1 and 2 , respectively.

\section{Blood pressure}

SBP exhibited time $(P \leq 0 \cdot 01)$ and treatment $\times$ time interaction effects $(P=0.002)$. A post hoc Šidák test indicated that this difference occurred at 1,2,3 h after supplementation in the MC group, with peak reductions from baseline in postprandial SBP of $6 \pm 2 \mathrm{mmHg}$ at $1 \mathrm{~h}$ after MC consumption (Fig. 1). There were no time, treatment or treatment $\times$ time interaction effects observed for diastolic BP.

\section{Transcranial Doppler imaging}

There was no time, treatment or treatment $\times$ time interaction effects observed for CBFV $(P>0.05)$.

\section{Near-IR spectroscopy parameters}

Oxygenated $H b$. Similarly, there was a significant interaction between treatment and post-treatment epoch on the initial ANOVA during the resting/absorption period $(P=0 \cdot 029)$. Reference to planned comparisons showed that there were significantly higher oxy-Hb concentrations during the 30-40-min epoch of the resting/absorption period for MC concentrate. The $\mathrm{MC}$ concentrate also resulted in higher oxy-Hb concentrations during each epoch of task performance $1 \mathrm{~h}$ after consumption $(P=0 \cdot 019)$. Thereafter, there were no significant differences in oxy-Hb $(P>0 \cdot 05)$ (Fig. 2(a)).

Deoxygenated $\mathrm{Hb}$. There were no significant differences in terms of deoxy-Hb during either the resting/absorption period or the task performance periods $(P>0 \cdot 05)$

Total $H b$. There was no significant interaction between treatment and post-treatment epoch on the initial ANOVA during the resting/absorption period $(P>0 \cdot 05)$. The MC concentrate resulted in higher total $\mathrm{Hb}$ concentrations during each epoch of task performance $1 \mathrm{~h}$ after consumption $(P \leq 0 \cdot 01)$. Thereafter, there were no significant differences in total $\mathrm{Hb}(P>0.05)$ (Fig. 2(b)).

Task-related differences. There were no significant differences seen in the haemodynamic response to the DV, RVIP or Stroop tasks.

\section{Discussion}

To the best of our knowledge, this study was the first to investigate the acute effects of $\mathrm{MC}$ consumption on $\mathrm{CBF}$ variables and cognitive performance in a middle-aged population. In support of our hypothesis, this study presents new information that, in comparison with placebo, consumption of a $\mathrm{MC}$ concentrate resulted in acute modulation of CBF parameters in the frontal cortex during task performance as indicated by the

Table 1. Effects of Montmorency tart cherry (MC) concentrate and placebo (PLA) on various aspects of cognitive performance in healthy, middle-aged adults (Mean values with their standard errors; $n$ 27)

\begin{tabular}{|c|c|c|c|c|c|c|c|c|c|c|c|c|c|c|}
\hline \multirow[b]{3}{*}{ Measures } & \multirow[b]{3}{*}{ Treatments } & \multicolumn{10}{|c|}{ Task battery repetition } & & & \\
\hline & & \multicolumn{2}{|c|}{ Baseline } & \multicolumn{2}{|c|}{1} & \multicolumn{2}{|c|}{2} & \multicolumn{2}{|c|}{3} & \multicolumn{2}{|c|}{5} & \multicolumn{3}{|c|}{ ANOVA } \\
\hline & & Mean & SEM & Mean & SEM & Mean & SEM & Mean & SEM & Mean & SEM & Effect & $F$ & $P$ \\
\hline \multirow[t]{2}{*}{ DV (\%) } & $60 \mathrm{ml} \mathrm{MC}$ & $94 \cdot 20$ & $1 \cdot 11$ & $94 \cdot 30$ & 1.40 & 93.58 & 1.38 & $92 \cdot 29$ & 1.67 & $92 \cdot 28$ & 2.00 & $\mathrm{~T}$ & 0.087 & 0.771 \\
\hline & PLA & $95 \cdot 17$ & 0.88 & 94.47 & 1.16 & 94.08 & 1.33 & 92.59 & 1.78 & $92 \cdot 10$ & 2.02 & $T \times R$ & 0.137 & 0.890 \\
\hline \multirow[t]{2}{*}{ DV RT (ms) } & $60 \mathrm{ml} \mathrm{MC}$ & $455 \cdot 41$ & 8.08 & 461.85 & 8.99 & 464.01 & 8.01 & $472 \cdot 10$ & 8.93 & 470.05 & 9.35 & $T$ & 3.793 & 0.062 \\
\hline & PLA & 455.48 & 8.19 & 461.02 & 8.58 & $465 \cdot 76$ & 9.10 & 454.59 & 11.06 & 443.96 & $16 \cdot 15$ & $T \times R$ & $2 \cdot 109$ & 0.135 \\
\hline \multirow[t]{2}{*}{ RVIP (\%) } & $60 \mathrm{ml} \mathrm{MC}$ & 53.40 & 5.04 & 52.85 & 4.23 & $51 \cdot 24$ & 5.08 & $51 \cdot 70$ & 4.98 & $52 \cdot 31$ & 4.80 & $\mathrm{~T}$ & 0.027 & 0.870 \\
\hline & PLA & 51.69 & 4.30 & $55 \cdot 12$ & 4.67 & 51.34 & 4.87 & 53.47 & 4.47 & $52 \cdot 15$ & 4.73 & $T \times R$ & 0.391 & 0.759 \\
\hline \multirow[t]{2}{*}{ RVIP RT (ms) } & $60 \mathrm{ml} \mathrm{MC}$ & 491.55 & 32.22 & 517.05 & $12 \cdot 13$ & $522 \cdot 39$ & 10.99 & 527.96 & 10.99 & 504.35 & 11.81 & $\mathrm{~T}$ & 0.269 & 0.608 \\
\hline & PLA & $526 \cdot 14$ & 11.55 & 520.01 & 12.02 & 483.81 & 29.07 & 505.52 & $17 \cdot 11$ & 506.96 & 15.74 & $T \times R$ & $1 \cdot 145$ & 0.316 \\
\hline \multirow{2}{*}{ Stroop (\%) } & $60 \mathrm{ml} \mathrm{MC}$ & 98.65 & 0.24 & $98 \cdot 78$ & 0.26 & 98.69 & 0.24 & 98.77 & 0.23 & 98.62 & 0.25 & T & 0.960 & 0.414 \\
\hline & PLA & 98.58 & 0.24 & 98.65 & 0.30 & 98.89 & 0.24 & 99.01 & 0.20 & 98.96 & 0.21 & $T \times R$ & 0.667 & 0.298 \\
\hline \multirow[t]{2}{*}{ Stroop RT (ms) } & $60 \mathrm{ml} \mathrm{MC}$ & 789.04 & 30.74 & 774.62 & $26 \cdot 85$ & 778.11 & 36.00 & 753.95 & $26 \cdot 71$ & 761.07 & 24.48 & $\mathrm{~T}$ & 0.214 & 0.648 \\
\hline & PLA & 814.87 & 37.53 & 764.02 & $29 \cdot 23$ & 764.00 & 29.23 & $763 \cdot 36$ & 29.75 & $805 \cdot 29$ & $68 \cdot 20$ & $\mathrm{~T} \times \mathrm{R}$ & 0.677 & 0.487 \\
\hline
\end{tabular}

DV, digit vigilance; T, treatment; R, repetition; RT, reaction time; RVIP, rapid visual information processing. 
Table 2. Effects of Montmorency tart cherry (MC) concentrate and placebo (PLA) on mood in healthy, middle-aged subjects (Mean values with their standard errors; $n$ 27)

\begin{tabular}{|c|c|c|c|c|c|c|c|c|c|c|c|c|c|c|}
\hline \multirow[b]{3}{*}{ Measures } & \multirow[b]{3}{*}{ Treatment } & \multicolumn{10}{|c|}{ Task battery repetition } & & & \\
\hline & & \multicolumn{2}{|c|}{ Baseline } & \multicolumn{2}{|c|}{1} & \multicolumn{2}{|c|}{2} & \multicolumn{2}{|c|}{3} & \multicolumn{2}{|c|}{5} & \multicolumn{3}{|c|}{ ANOVA } \\
\hline & & Mean & SEM & Mean & SEM & Mean & SEM & Mean & SEM & Mean & SEM & Effect & $F$ & $P$ \\
\hline \multirow[t]{2}{*}{ Alert } & $60 \mathrm{ml} \mathrm{MC}$ & $35 \cdot 67$ & 4.55 & $35 \cdot 39$ & 3.55 & $40 \cdot 31$ & $4 \cdot 11$ & 42.91 & 4.41 & $45 \cdot 22$ & 4.44 & $\mathrm{~T}$ & 0.415 & 0.525 \\
\hline & PLA & $35 \cdot 10$ & 3.91 & $34 \cdot 76$ & 3.49 & $43 \cdot 13$ & $3 \cdot 70$ & $45 \cdot 30$ & 3.94 & $49 \cdot 85$ & $4 \cdot 37$ & $T \times R$ & 0.763 & 0.477 \\
\hline \multirow[t]{2}{*}{ Concentration } & $60 \mathrm{ml} \mathrm{MC}$ & $57 \cdot 19$ & 4.83 & 52.50 & 3.68 & $52 \cdot 39$ & 3.69 & $50 \cdot 94$ & 3.49 & $47 \cdot 96$ & 3.42 & $\mathrm{~T}$ & 0.287 & 0.597 \\
\hline & PLA & $51 \cdot 75$ & $3 \cdot 61$ & $57 \cdot 02$ & 3.19 & $48 \cdot 80$ & 3.44 & $49 \cdot 15$ & 3.50 & $46 \cdot 93$ & 3.52 & $T \times R$ & 1.417 & 0.250 \\
\hline \multirow[t]{2}{*}{ Mental fatigue } & $60 \mathrm{ml} \mathrm{MC}$ & $60 \cdot 74$ & 4.29 & 61.54 & 3.76 & 60.94 & $4 \cdot 13$ & 58.69 & 3.97 & $57 \cdot 20$ & $4 \cdot 21$ & $\mathrm{~T}$ & 0.163 & 0.690 \\
\hline & PLA & 61.65 & 3.95 & 63.44 & $3 \cdot 32$ & $55 \cdot 76$ & 3.82 & $54 \cdot 30$ & 4.09 & $56 \cdot 85$ & 4.08 & $T \times R$ & 1.281 & 0.288 \\
\hline \multirow{2}{*}{ Difficulty } & $60 \mathrm{ml} \mathrm{MC}$ & 38.41 & 3.91 & 38.94 & 3.46 & 40.50 & $4 \cdot 21$ & 41.57 & $4 \cdot 21$ & 44.59 & 3.81 & $\mathrm{~T}$ & 0.014 & 0.907 \\
\hline & PLA & $40 \cdot 73$ & 3.69 & 36.65 & 3.41 & 39.78 & 3.08 & 38.96 & 3.43 & 44.96 & 3.50 & $T \times R$ & 0.631 & 0.579 \\
\hline
\end{tabular}

$\mathrm{T}$, treatment; $\mathrm{R}$, repetition.

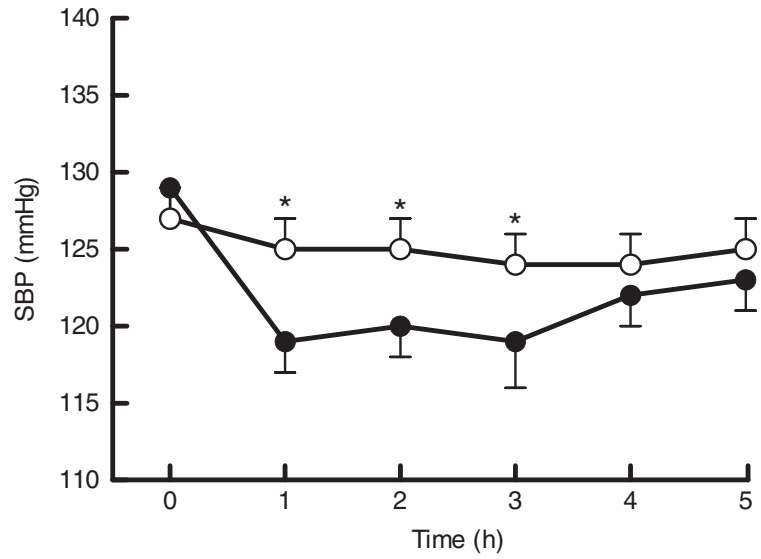

Fig. 1. Time course of systolic blood pressure (SBP) response after consumption of Montmorency tart cherry (MC) concentrate and macronutrient-matched control. Values are means $(n 27)$, with their standard errors. The mean value was significantly different from that of the placebo drink: ${ }^{*} P<0.05 .-, 60 \mathrm{ml} \mathrm{MC} ; \multimap-$, placebo.

elevated concentration of total $\mathrm{Hb}$, with an identical pattern observed with oxy-Hb. This effect was evident for the cognitive assessment $1 \mathrm{~h}$ after MC consumption. These CBF observations were not associated with any significant modulation of cognitive performance or mood. There was also a significant reduction in SBP for up to $3 \mathrm{~h}$ after MC consumption relative to the placebo.

Compromised CBF has been suggested as a key contributor to cognitive function decline observed with advancing age and in a number of neurodegenerative diseases ${ }^{(46)}$. The results of the present study demonstrate that MC concentrate can modulate aspects of brain function, which was evident $1 \mathrm{~h}$ after consumption. Total $\mathrm{Hb}$ and oxy-Hb were increased towards the end of the 60-min resting/absorption period, although not significantly, in most cases, and during the cognitive assessment $1 \mathrm{~h}$ after consumption. However, there were no concomitant changes in deoxy-Hb across any of the time points. These results are consistent with previous studies using compounds and whole foods to demonstrate a positive effect on cognitive function and CBF. Kennedy et $a l{ }^{(30)}$ demonstrated an increase in total $\mathrm{Hb}$ and oxy- $\mathrm{Hb}$ following single doses of orally

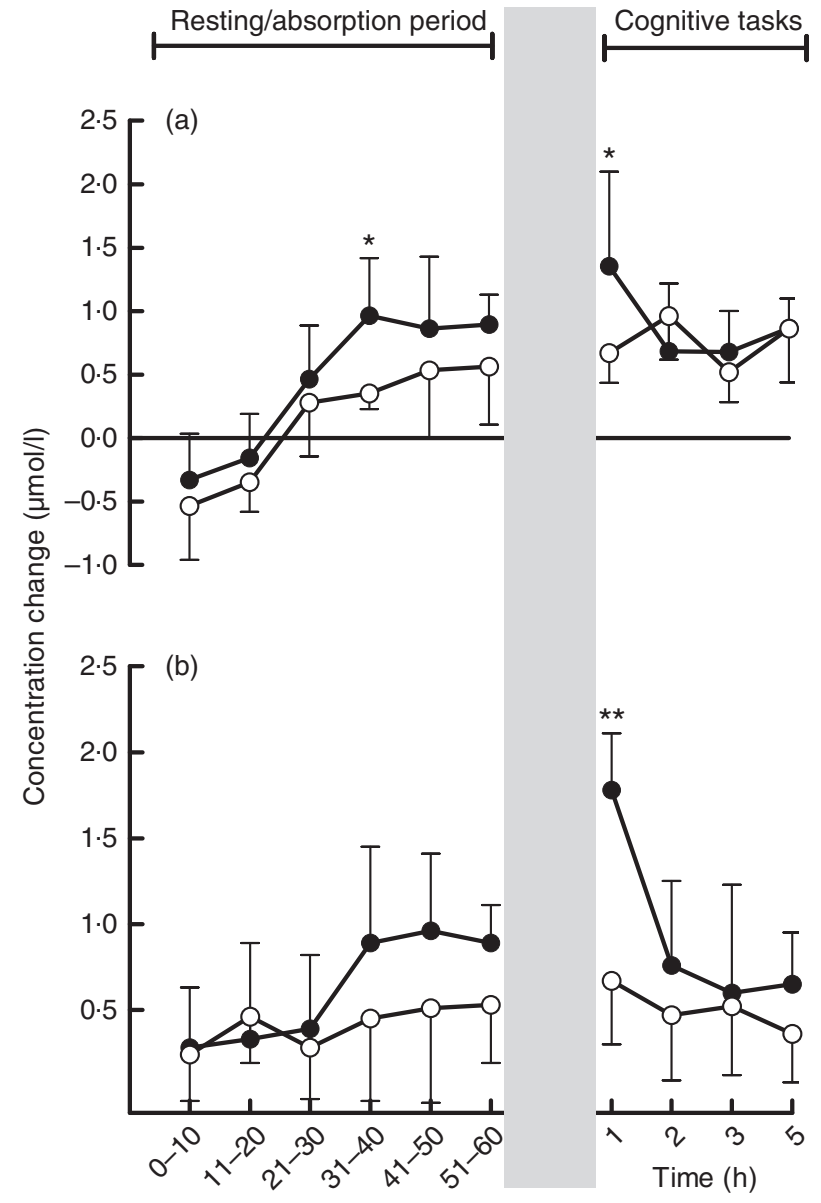

Fig. 2. (a) Mean changes in concentrations of oxygenated $\mathrm{Hb}$ and (b) total $\mathrm{Hb}$ during a 60-min absorption period and subsequent cognitive task assessments $1,2,3$ and $5 \mathrm{~h}$ after consumption of $60-\mathrm{ml}$ Montmorency tart cherry (MC) concentrate or placebo. Values are means with their standard errors. The mean value was significantly different from that of the placebo: ${ }^{*} P<0.05$, ${ }^{* *} P<0.01$. - - $60 \mathrm{ml} \mathrm{MC} ;-\mathrm{O}-$, placebo.

administered resveratrol, and more recently Wightman et al. ${ }^{(31)}$ demonstrated an increase following beetroot juice ingestion. In both of these studies, total $\mathrm{Hb}$ was increased during the first epoch of task performance. However, although total $\mathrm{Hb}$ 
remained higher in the resveratrol study throughout the 40-min cognitive assessment, it was decreased during the last five repetitions when participants were supplemented with beetroot juice. In the present study, no significant differences were seen following the first task period. The limitations associated with NIRS have consistently been highlighted ${ }^{(47)}$, and in the past few years fMRI and other neuroimaging techniques have been used to assess the effect of a nutritional supplement on $\mathrm{CBF}^{(33,48)}$.

In terms of higher total $\mathrm{Hb}$ concentrations, the modulation seen in the present study may be due to the vasorelaxatory and antihypertensive properties of some of the phenolic acids (vanillic and protocatechuic acid) contained in the MC concentrate $^{(3,10)}$. The time points (approximately $1 \mathrm{~h}$ after) at which these metabolites are seen in the plasma coincide with improvements in vascular function ${ }^{(10)}$ and modulation in CBF in the present study. Although deoxy-Hb was not modulated by the experimental beverage, it should be noted that there was a trend for a reduced concentration throughout the task period. Furthermore, in the present study, task had no significant effect on cerebral modulation. It has been previously speculated that 'high activation' tasks such as RVIP result in a higher increase in CBF compared with performance in 'low activation' tasks - for example, DV. This can be largely attributed to the relative cognitive demands of the two tasks, with RVIP requiring the monitoring of rapidly changing digits along with a passive contribution from working memory. We speculate that these very early on effects on CBF in the present study are more likely to be associated with the sensory properties of the MC concentrate, as previous studies have demonstrated that a number of sensory factors including differing taste and flavours are likely to modulate frontal cortex activity ${ }^{(49,50)}$. Marciani et al. have previously demonstrated that several brain areas were activated immediately after swallowing, particularly when supplements had a strong (combined) taste or aroma. It could be argued that the MC concentrate was more sensory stimulating than the placebo. However, a full analysis of sensory properties was outside the remit of the present study.

Although the present study highlights an acute heightened NIRS response in brain regions responsible for task performance, there was no effect on cognitive performance. Supplementary $\mathrm{O}^{(51)}$ has been shown to positively influence cognitive performance in a healthy population. Therefore, it makes the expectation tenable that increases in CBF could be beneficial to acute cognitive performance via increasing the delivery of oxygenated blood metabolic substrate to and efflux of metabolites from the brain, which is critical for brain function ${ }^{(52)}$. Importantly, despite some indication of improved blood flow, the present study showed no changes in cognitive task performance between experimental conditions. Nevertheless, these results do not stand alone; Caldwell et al. ${ }^{(24)}$ previously demonstrated that regardless of dose, cherry juice had no acute impact on cognitive function in young people, older people or dementia patients. They concluded that, although cherry juice may have an acute impact on cardiovascular function, there was no change in cognitive performance $6 \mathrm{~h}$ after consumption. However, Caldwell et al. used sweet cherries for the intervention, and it has been speculated that sweet cherries are not as rich in phytochemical compounds as tart/sour cherries ${ }^{(6)}$. Furthermore, cognitive assessments and blood flow measures were taken at baseline, 2 and $6 \mathrm{~h}$ after consumption, and the present study attempted to explore the time points following consumption in more detail (hourly); however, it is possible that some potential changes might still have been missed. Contrastingly, a chronic study by the same group ${ }^{(25)}$ reported that daily consumption of sweet cherries for 12 weeks improved cognitive performance across almost all tasks in older adults with mild-to-moderate dementia; this group showed improvements for category verbal fluency and tasks relating to verbal learning and memory and concluded that the positive changes have clinical relevance for these cognitive improvements. Therefore, it is likely that regulation of blood flow and cognition are extremely complex, with multiple overlapping regulatory mechanisms, paradigms and contributing structural components ${ }^{(53)}$, and therefore more likely to be influenced by chronic supplementation. This also accords with previous observations in similar trials, where Kelly et $a l .{ }^{(54)}$ and Thompson et al. ${ }^{(55)}$ showed that after acute beetroot supplementation there were no changes in cognitive performance for concentration, memory, attention or information processing ability. However, when older type 2 diabetics were supplemented with beetroot juice for $14 \mathrm{~d}$, they experienced a significant improvement in simple reaction time compared with a control group ${ }^{(56)}$. These somewhat contrasting results may be partly explained by dose duration. It would seem that the cerebrovascular response required to elicit measurable changes in cognitive function can only be achieved with longer-term dosing strategies that have the potential to induce sustained modifications to cerebrovascular function ${ }^{(26)}$. However, contrary to this suggestion, two recent additions to the literature have demonstrated positive effects on cognitive function following acute blackcurrant $^{(27)}$ and $\mathrm{WBB}^{(28)}$ supplementation. Acute blackcurrant supplementation was shown to improve RVIP accuracy and reaction time on the DV task in healthy people; however, Whyte et al. demonstrated that acute cognitive benefits can be observed in 7-10-year old children with an anthocyanin-rich blueberry intervention. The Page's test revealed the consistency and strength of this finding with WBB supplementation, leading to significant overall improvements in cognition function, with the best change from baseline performance associated with $30 \mathrm{~g}$ WBB treatment, intermediate performance with the $15 \mathrm{~g}$ WBB treatment and the least effective performance with the vehicle treatment. Given that the protective cognitive effect of fruits is attributed to their high anthocyanin content, the anthocyanin dose in both of these studies was marginally higher compared with the current investigation (253 and $552 \mathrm{mg} v$. $68 \mathrm{mg}$ cyanidin-3-glucoside/1), this might go some way in explaining the inconsistent findings. It is also worthy to note that the cognitive tasks for the current study were selected on the basis of previous sensitivity to nutritional interventions ${ }^{(18,27)}$; however, these tasks may not be adequately sensitive to detect change in acute studies, as perhaps this particular intervention could affect different cognitive domains (i.e. memory, recall). The most important consideration in setting up a suitable framework for measuring human cognitive function in polyphenol or flavonoid research is to determine methods that are sensitive to 
dietary changes and repeatable over time, simple to interpret and specific to cognitive domains ${ }^{(57)}$. Furthermore, all participants in the present study were healthy with no apparent issues pertaining to $\mathrm{CBF}$ or cognitive ability. It is logical to question whether this means that sufficient blood flow already exists for maximal cognitive performance, and therefore increasing blood flow beyond this threshold does not have any acute benefits on cognitive performance.

In addition, there was no effect of the intervention on mood. This is somewhat surprising as mental fatigue has been previously shown to be receptive to cocoa flavanols in healthy adults $^{(18)}$. However, Scholey et al. used repeated 10-min cycles of a cognitive demand battery (two serial subtraction tasks (serial threes and serial sevens) and RVIP), over the course of $1 \mathrm{~h}$. Therefore, the cognitive assessment adopted in the present study might not have been as taxing on the brain as the aforementioned cognitive demand battery with very limited rest time between repetitions.

There was a significant decrease in SBP following MC supplementation when compared with placebo. These findings are in agreement with a previous study that reported a positive modulation of SBP in early hypertensive males following MC ingestion $^{(10)}$. This is not surprising, as participants in the present study had moderately elevated SBP above the published ideal values at baseline $-128 / 82 \mathrm{mmHg}$. Kapil et al ${ }^{(58)}$ noted that the magnitude of change in the BP response is directly related to baseline BP; therefore, those who have a higher BP will likely experience a greater change following an intervention. The present study is particularly noteworthy as data from prospective, observational studies have shown that a reduction in mean SBP of $5-6 \mathrm{mmHg}$ over a 5 -year period was associated with 38 and $23 \%$ reduced risk of stroke and CHD, respectively ${ }^{(59)}$. In this study, we reported peak reductions in postprandial SBP of $6 \pm 2 \mathrm{mmHg}$ relative to the placebo. This finding, along with the modulation of $\mathrm{CBF} 1 \mathrm{~h}$ after MC consumption, supports the growing body of evidence showing an inverse association between the risk of chronic human diseases and the consumption of polyphenolic-rich diets ${ }^{(60,61)}$

The findings of the present study should be interpreted with a certain degree of caution because of the dietary restrictions imposed on participants. It is extremely unlikely that one would consume a diet that is free from polyphenol-rich foods, and as a result future studies should attempt to demonstrate synergistic effects of MC supplementation within habitual dietary practices. Furthermore, a digital automatic BP monitor was used in the present study. The accuracy of this method has been called into question $^{(62)}$. Future studies should consider using ambulatory $\mathrm{BP}$ measurements, where readings are taking at regular intervals. Many studies have now confirmed that BP measured over a 24-h period is superior to clinic BP in predicting future cardiovascular events ${ }^{(63)}$. A timeframe of $5 \mathrm{~h}$ was utilised on the basis of previous findings that phenolic compounds are quickly absorbed and/or excreted ${ }^{(3,36)}$ and that any positive effects in vascular function are transient and return to baseline after $4 \mathrm{~h}^{(10)}$. However, it is possible that this timeframe may not have been long enough to capture the absorption of potentially other bioactive phenolic compounds provided by cherries in the colon.
In summary, the findings of this study suggest that MC concentrate can acutely modulate $\mathrm{CBF}$ in the prefrontal cortex, characterised by increased concentrations of both total $\mathrm{Hb}$ and oxy-Hb. Despite this evident modulation, these results do not translate to improvements in cognition or mood in the hours following consumption. Finally, this study re-affirms previous findings that demonstrate a significant improvement in SBP following MC supplementation.

\section{Acknowledgements}

The authors thank the participants of this investigation.

The Cherry Research Committee of the Cherry Marketing Institute (Lansing, MI, USA), a not-for-profit organisation, provided support for a $\mathrm{PhD}$ studentship associated with this study. All other elements of the study were funded by Northumbria University. The funders had no role in the study design, data collection and analysis, decision to publish or preparation of the manuscript.

The authors' responsibilities were as follows: K. M. K., R. C. V., C. F. H.-R. and G. H. designed the study; K. M. K. conducted the study; K. M. K. G. H. and C. F. H.-R. analysed and interpreted the data; K. M. K., C. F. H.-R. and G. H. wrote the paper; G. H. and K. M. K. had primary responsibility for the final content. All authors read and approved the final manuscript.

The authors declare that there are no conflicts of interest.

\section{References}

1. Wang HB, Nair MG, Strasburg GM, et al. (1999) Antioxidant and antiinflammatory activities of anthocyanins and their aglycon, cyanidin, from tart cherries. J Nat Prod 62, 802-802.

2. Bell PG, Gaze DC, Davison GW, et al. (2014) Montmorency tart cherry (Prunus cerasus L.) concentrate lowers uric acid, independent of plasma cyanidin-3-O-glucosiderutinoside. J Funct Foods 11, 82-90.

3. Keane KM, Bell PG, Lodge JK, et al. (2016) Phytochemical uptake following human consumption of Montmorency tart cherry (L. Prunus cerasus) and influence of phenolic acids on vascular smooth muscle cells in vitro. Eur J Nutr $\mathbf{5 5}$, $1695-1705$.

4. Seeram NP, Momin RA, Nair MG, et al. (2001) Cyclooxygenase inhibitory and antioxidant cyanidin glycosides in cherries and berries. Phytomedicine 8, 362-369.

5. Seymour EM, Warber SM, Kirakosyan A, et al. (2014) Anthocyanin pharmacokinetics and dose-dependent plasma antioxidant pharmacodynamics following whole tart cherry intake in healthy humans. J Funct Foods 11, 509-516.

6. Kim DO, Heo HJ, Kim YJ, et al. (2005) Sweet and sour cherry phenolics and their protective effects on neuronal cells. J Agric Food Chem 53, 9921-9927.

7. Kirakosyan A, Seymour EM, Llanes DEU, et al. (2009) Chemical profile and antioxidant capacities of tart cherry products. Food Chem 115, 20-25.

8. Bell PG, Walshe IH, Davison GW, et al. (2014) Montmorency cherries reduce the oxidative stress and inflammatory responses to repeated days high-intensity stochastic cycling. Nutrients 6, 829-843.

9. Howatson G, McHugh MP, Hill JA, et al. (2010) Influence of tart cherry juice on indices of recovery following marathon running. Scand J Med Sci Sports 20, 843-852. 
10. Keane KM, George TW, Constantinou CL, et al. (2016) Effects of Montmorency tart cherry (Prunus cerasus L.) consumption on vascular function in men with early hypertension. $A m$ J Clin Nutr 103, 1531-1539.

11. Marchal G, Rioux P, Petittaboue MC, et al. (1992) Regional cerebral oxygen-consumption, blood-flow, and blood-volume in healthy-human aging. Arch Neurol 49, 1013-1020.

12. Joseph JA, Bartus RT, Clody D, et al. (1983) Psychomotor performance in the senescent rodent - reduction of deficits via striatal dopamine receptor up-regulation. Neurobiol Aging $\mathbf{4}$, 313-319.

13. Shukitt-Hale B, Mouzakis G \& Joseph JA (1998) Psychomotor and spatial memory performance in aging male Fischer 344 rats. Exp Gerontol 33, 615-624.

14. Hofer SM, Berg S \& Era P (2003) Evaluating the interdependence of aging-related changes in visual and auditory acuity, balance, and cognitive functioning. Psychol Aging 18, 285-305.

15. Ajmani RS, Metter EJ, Jaykumar R, et al. (2000) Hemodynamic changes during aging associated with cerebral blood flow and impaired cognitive function. Neurobiol Aging 21, 257-269.

16. Chan YC, Hosoda K, Tsai CJ, et al. (2006) Favorable effects of tea on reducing the cognitive deficits and brain morphological changes in senescence-accelerated mice. J Nutr Sci Vitaminol 52, 266-273.

17. Birks J \& Evans JG (2009) Ginkgo biloba for cognitive impairment and dementia. The Cochrane Database of Systematic Reviews, no. 1, Article ID CD003120.

18. Scholey AB, French SJ, Morris PJ, et al. (2010) Consumption of cocoa flavanols results in acute improvements in mood and cognitive performance during sustained mental effort. J Psychopharmacol 24, 1505-1514.

19. Shukitt-Hale B, Lau FC \& Joseph JA (2008) Berry fruit supplementation and the aging brain. J Agric Food Chem $\mathbf{5 6}$ 636-641.

20. Shukitt-Hale B, Carey A, Simon L, et al. (2006) Effects of Concord grape juice on cognitive and motor deficits in aging. Nutrition 22, 295-302.

21. Seymour EM, Wolforth J, Bosak K, et al. (2013) Effect of tart cherry versus PPAR agonist pioglitazone on stroke-related phenotypes and inflammation. Experimental Biology. Boston, MA, 23 April 2013

22. Kirakosyan A, Seymour EM, Wolforth J, et al. (2015) Tissue bioavailability of anthocyanins from whole tart cherry in healthy rats. Food Chem 171, 26-31.

23. Thangthaeng NP, Gomes SM, Miller SM, et al. (2016) Tart cherry supplementation improves working memory, hippocampal inflammation, and autophagy in aged rats. Age (Dordr) (Epublication ahead of print version 30 August 2016).

24. Caldwell K, Charlton KE, Roodenrys S, et al. (2016) Anthocyanin-rich cherry juice does not improve acute cognitive performance on RAVLT. Nutr Neurosci 19, 423-424.

25. Kent K, Charlton K, Roodenrys S, et al. (2015) Consumption of anthocyanin-rich cherry juice for 12 weeks improves memory and cogntion in older adults with mild-to-moderate dementia. Eur J Nutr (epublication ahead of print version 19 October 2015).

26. Clifford T, Howatson G, West DJ, et al. (2015) The potential benefits of red beetroot supplementation in health and disease. Nutrients 7, 2801-2822.

27. Watson AW, Haskell-Ramsay CF, Kennedy DO, et al. (2015) Acute supplementation with blackcurrant extracts modulates cognitive functioning and inhibits monoamine oxidase- $\mathrm{B}$ in healthy young adults. J Funct Foods 17, 524-539.
28. Whyte AR, Schafer G \& Williams CM (2016) Cognitive effects following acute wild blueberry supplementation in 7 - to 10-year-old children. Eur J Nutr 55, 2151-2162.

29. Wightman EL, Haskell CF, Forster JS, et al. (2012) Epigallocatechin gallate, cerebral blood flow parameters, cognitive performance and mood in healthy humans: a double-blind, placebo-controlled, crossover investigation. Hum Psychopharmacol 27, 177-186.

30. Kennedy DO, Wightman EL, Reay JL, et al. (2010) Effects of resveratrol on cerebral blood flow variables and cognitive performance in humans: a double-blind, placebo-controlled, crossover investigation. Am J Clin Nutr 91, 1590-1597.

31. Wightman EL, Haskell-Ramsay CF, Thompson KG, et al. (2015) Dietary nitrate modulates cerebral blood flow parameters and cognitive performance in humans: a double-blind, placebo-controlled, crossover investigation. Physiol Behav 149, 149-158.

32. Krikorian R, Boespflug EL, Fleck DE, et al. (2012) Concord grape juice supplementation and neurocognitive function in human aging. J Agric Food Chem 60, 5736-5742.

33. Bookheimer SY, Renner BA, Ekstrom A, et al. (2013) Pomegranate juice augments memory and fMRI activity in middle-aged and older adults with mild memory complaints. Evid Based Complement Alternat Med 2013, 946298.

34. Francis ST, Head K, Morris PG, et al. (2006) The effect of flavanol-rich cocoa on the fMRI response to a cognitive task in healthy young people. J Cardiovasc Pharmacol 47, S215-S220.

35. Sorond FA, Lipsitz LA, Hollenberg NK, et al. (2008) Cerebral blood flow response to flavanol-rich cocoa in healthy elderly humans. Neurophychiatr Dis Treat 4, 433-440.

36. Manach C, Williamson G, Morand C, et al. (2005) Bioavailability and bioefficacy of polyphenols in humans. I. Review of 97 bioavailability studies. Am J Clin Nutr 81, 230S-242S.

37. Dodd FL, Kennedy DO, Riby LM, et al. (2015) A double-blind, placebo-controlled study evaluating the effects of caffeine and L-theanine both alone and in combination on cerebral blood flow, cognition and mood. Psychopharmacology 232, 2563-2576.

38. Stonehouse W, Conlon CA, Podd J, et al. (2013) DHA supplementation improved both memory and reaction time in healthy young adults: a randomized controlled trial. Am J Clin Nutr 97, 1134-1143.

39. Drummond SPA, Brown GG, Stricker JL, et al. (1999) Sleep deprivation-induced reduction in cortical functional response to serial subtraction. Neuroreport 10, 3745-3748.

40. Lawrence NS, Ross TJ \& Stein EA (2002) Cognitive mechanisms of nicotine on visual attention. Neuron 36, 539-548.

41. Coull JT, Frith CD, Frackowiak RSJ, et al. (1996) A frontoparietal network for rapid visual information processing: a PET study of sustained attention and working memory. Neuropsychologia 34, 1085-1095.

42. Homack S \& Riccio CA (2004) A meta-analysis of the sensitivity and specificity of the Stroop Color and Word Test with children. Arch Clin Neuropsychol 19, 725-743.

43. Aaslid R, Markwalder TM \& Nornes H (1982) Non-invasive transcranial Doppler ultrasound recording of flow velocity in basal cerebral arteries. J Neurosurg 57, 769-774.

44. Schecklmann M, Ehlis A-C, Plichta MM, et al. (2008) Functional near-infrared spectroscopy: a long-term reliable tool for measuring brain activity during verbal fluency. Neuroimage 43, 147-155.

45. Wightman EL, Reay JL, Haskell CF, et al. (2014) Effects of resveratrol alone or in combination with piperine on cerebral blood flow parameters and cognitive performance in human subjects: a randomised, double-blind, placebo-controlled, cross-over investigation. Br J Nutr 112, 203-213. 
46. Farkas E, de Wilde MC, Kiliaan AJ, et al. (2002) Chronic cerebral hypoperfusion-related neuropathologic changes and compromised cognitive status: window of treatment. Drugs Today 38, 365-376.

47. Murkin JM \& Arango M (2009) Near-infrared spectroscopy as an index of brain and tissue oxygenation. Br J Anaesth 103, Suppl. 1, i3-i13.

48. Isaacs EB (2013) Neuroimaging, a new tool for investigating the effects of early diet on cognitive and brain development. Front Hum Neurosci 7, 445.

49. Marciani L, Pfeiffer JC, Hort J, et al. (2006) Improved methods for fMRI studies of combined taste and aroma stimuli. J Neurosci Methods 158, 186-194

50. Smits M, Peeters RR, van Hecke P, et al. (2007) A 3 T eventrelated functional magnetic resonance imaging (fMRI) study of primary and secondary gustatory cortex localization using natural tastants. Neuroradiology 49, 61-71.

51. Moss MC, Scholey AB \& Wesnes K (1998) Oxygen administration selectively enhances cognitive performance in healthy young adults: a placebo controlled double blind crossover study. Psychopharmacology 138, 27-33.

52. Attwell D, Buchan AM, Charpak S, et al. (2010) Glial and neuronal control of brain blood flow. Nature 468, 232-243.

53. Peterson EC, Wang Z \& Britz G (2011) Regulation of cerebral blood flow. Int J Vasc Med 2011, Article ID 823525.

54. Kelly J, Fulford J, Vanhatalo A, et al. (2013) Effects of short-term dietary nitrate supplementation on blood pressure, O-2 uptake kinetics, and muscle and cognitive function in older adults. $\mathrm{Am}$ J Physiol Regul Integr Comp Physiol 304, R73-R83.
55. Thompson KG, Turner L, Prichard J, et al. (2014) Influence of dietary nitrate supplementation on physiological and cognitive responses to incremental cycle exercise. Respir Physiol Neurobiol 193, 11-20.

56. Gilchrist M, Winyard PG, Fulford J, et al. (2014) Dietary nitrate supplementation improves reaction time in type 2 diabetes: development and application of a novel nitrate-depleted beetroot juice placebo. Nitric Oxide 40, 67-74.

57. Macready AL, Kennedy OB, Ellis JA, et al. (2009) Flavonoids and cognitive function: a review of human randomized controlled trial studies and recommendations for future studies. Genes Nutr 4, 227-242.

58. Kapil V, Milsom AB, Okorie M, et al. (2010) Inorganic nitrate supplementation lowers blood pressure in humans role for nitrite-derived NO. Hypertension 56, 274-281.

59. Collins R, Peto R, Macmahon S, et al. (1990) Blood-pressure, stroke, and coronary heart disease. 2 . Short-term reductions in blood-pressure - overview of randomized drug trials in their epidemiologic context. Lancet 335, 827-838.

60. Pandey KB \& Rizvi SI (2009) Plant polyphenols as dietary antioxidants in human health and disease. Oxid Med Cell Longev 2, 270-278.

61. Ginter E \& Simko V (2012) Plant polyphenols in prevention of heart disease. Bratisl Lek Listy 113, 476-480.

62. Nelson D, Kennedy B, Regnerus C, et al. (2008) Accuracy of automated blood pressure monitors. J Dental Hyg 84, 35.

63. Ogedegbe $\mathrm{G}$ \& Pickering $\mathrm{T}$ (2010) Principles and techniques of blood pressure measurement. Cardiol Clin 28, 571-586. 